\title{
Características clínicas asociadas a colitis eosinofílica en lactantes con rectorragia persistente
}

\author{
B. Romero・ E. Talesnik • J. Cerda ・ P. R. Harris
}

\author{
ABSTRACT \\ Clinical features of Eosinophilic Colitis in infants with persistent rectal bleeding
}

The most common presentation of cow's milk protein allergy (CMP) in infants is known as eosinophilic colitis (EC). The aim of this study is to evaluate EC characteristics in infants evaluated with colonoscopy due to the presence of rectorrhagia. Patients and Methods: A retrospective case-control study. Left-sided colonoscopy records of infants with persistent rectal bleeding, conducted between January 2006 and March 2011, were reviewed. The cases corresponded to infants with rectal biopsy compatible with EC and controls with negative biopsy. Telephone questionnaires to parents were conducted, evaluating personal and family history. Results: Complete records were obtained in $61(79 \%)$ of the 77 procedures. $33(54 \%)$ of them were males. Examination average age was $6.3 \pm 5.9$ months. $25(41 \%)$ patients had $\mathrm{EC}$ on their histology. Between cases and controls, no significant difference in gestational age, birth weight and gender, only regarding age at the time of rectal bleeding, were observed. There was also no difference in personal history regarding obstructive bronchitis, allergic rhinitis, family history of asthma, allergic rhinitis or other food allergies. Those who received artificial feeding did not presented greater risk of EC. The most common symptoms in the cases did not differ significantly from the controls. Conclusions: The prevalence of EC in the children studied was $40.9 \%$. Our results show that there are groups of patients with persistent rectal bleeding in which there is no personal or family history that helps diagnosing EC. An endoscopic study could be considered in these patients to establish a correct diagnosis of this condition, avoid unnecessary diets and not to delay the detection of other diseases.

(Key words: Food allergies, colitis, milk protein).

Rev Chil Pediatr 2014; 85 (6): 666-673

\section{RESUMEN}

En lactantes, la forma de presentación más común de la alergia a la proteína de la leche de vaca (PLV) es la colitis eosinofílica (CE). El objetivo de este trabajo es evaluar características clínicas asociadas a CE en lactantes evaluados con colonoscopía por la presencia de rectorragia. Pacientes y Método: Estudio caso-control,

Recibido el 13 de agosto de 2013. Última versión aceptada el 06 de mayo de 2014.

Bernardita Romero, Eduardo Talesnik, Paul R. Harris $(\bowtie)$

División de Pediatría, Facultad de Medicina, Pontificia Universidad Católica de Chile, Santiago, Chile.

E-mail: pharris@med.puc.cl

Jaime Cerda

Departamento de Salud Pública, Facultad de Medicina, Pontificia Universidad Católica de Chile, Santiago, Chile. 
retrospectivo. Se revisaron registros de colonoscopía izquierda de lactantes con rectorragia persistente, realizadas entre Enero 2006 y Marzo 2011. Casos fueron lactantes con rectorragia y biopsia compatible con CE y controles aquellos con biopsia negativa. Se realizó un cuestionario vía telefónica a los padres, evaluándose antecedentes personales y familiares. Resultados: En 61 (79\%) de 77 procedimientos se obtuvo registros completos. $33(54 \%)$ eran hombres. Edad promedio del examen fue 6,3 $\pm 5,9$ meses. $25(41 \%)$ pacientes presentaron CE en la histología. Sin diferencia significativa en edad gestacional, peso de nacimiento ni sexo, pero si en edad de presentación de la rectorragia, entre casos y controles. Tampoco hubo diferencia en antecedentes personales de bronquitis obstructivas, rinitis alérgica, ni antecedentes familiares de asma, rinitis alérgica u otras alergias alimentarias. Quienes recibieron lactancia artificial no tuvieron mayor riesgo de CE. Los síntomas más frecuentes en los casos no se diferenciaron significativamente de los controles. Conclusión: La prevalencia de CE en los niños estudiados fue de 40,9\%. Nuestros resultados muestran que hay grupos de pacientes con rectorragia persistente en los cuales no existen antecedentes de la historia familiar ni personal que permitan establecer el diagnóstico de CE. Es en estos pacientes en los cuales podría considerarse el estudio endoscópico para establecer un correcto diagnóstico de esta patología, evitar dietas innecesarias y no retrasar la detección de otras enfermedades.

(Palabras clave: Alergia alimentaria, colitis, proteína láctea, alergia a la proteína de vaca).

Rev Chil Pediatr 2014; 85 (6): 666-673

\section{Introducción}

Durante los primeros años de vida, el principal antígeno alimentario es la leche de vaca. Esta contiene varias proteínas inmunogénicas, de las cuales la betalactoglobulina es la más comúnmente asociada a alergia alimentaria ${ }^{1}$. La reacción alérgica puede ser mediada por $\mathrm{IgE}$, sin embargo, el principal mecanismo de acción es multifactorial (células $\mathrm{T}$, mastocitos, eosinófilos, etc.). Esta respuesta inmune compleja puede resultar en la aparición más tardía de los síntomas ${ }^{2,3}$.

Se estima que la prevalencia de alergia a la proteína de leche de vaca (APLV) es de $2-7,5 \%$ en lactantes alimentados con fórmulas y de $0,5 \%$ en aquellos que reciben lactancia materna exclusiva ${ }^{4-6}$.

La APLV se manifiesta durante las primeras semanas de vida, estando presente los síntomas gastrointestinales o cutáneos en un $50 \%$ y respiratorios en un $20 \%{ }^{6,7}$. Dentro de los síntomas gastrointestinales se encuentran los cólicos, la diarrea y rectorragia. Esta última es común en aquellos pacientes que cursan con una colitis eosinofílica (CE), sin embargo, puede estar asociada a causas infecciosas, lesiones anatómicas, vasculares o colitis inespecíficas $^{8,9}$. En casos excepcionales la rectorragia puede corresponder al inicio de una enfermedad inflamatoria intestinal ${ }^{10}$. Se han estable- cido relaciones también entre APLV y reflujo persistente o constipación, sin embargo, esto no es claro.

El Gold Standard para el diagnóstico de APLV es el test de provocación oral, idealmente doble ciego ${ }^{11-13}$. Sin embargo, la realización del doble ciego es difícil de practicar, por lo cual puede considerarse el realizar pruebas con ciego simple. Estas pruebas no están indicadas en caso de anafilaxia.

Los hallazgos endoscópicos, eosinofilia periférica, niveles de IgE totales y específicos, así como los test cutáneos, presentan sensibilidades y especificidades variables y bajas. En los casos en que resulta comprometido el colon, la biopsia endoscópica muestra un infiltrado inflamatorio agudo, con eosinófilos en el epitelio y en la lámina propia mayor a 60 por 10 campos de mayor aumento (CMA $)^{4,14-17}$.

No existen estudios nacionales de prevalencia de APLV, sin embargo, al existir dificultad en el diagnóstico, parece existir un sobrediagnóstico de esta. Es por esto, que el objetivo de este estudio fue estimar la prevalencia de $\mathrm{CE}$ en un grupo de lactantes referidos por sus médicos gastroenterólogos tratantes a colonoscopia como estudio de rectorragia persistente o recurrente y determinar predictores clínicos (personales y familiares) para el desarrollo de $\mathrm{CE}$ en este grupo de pacientes. Esto nos permitirá acercarnos a un mejor diagnóstico clí- 
nico de esta patología y así evitar someter a lactantes y a sus madres a dietas de exclusión innecesarias y costosas.

\section{Pacientes y Método}

\section{Diseño del estudio}

Estudio caso-control, retrospectivo. Se estudió un grupo de pacientes menores de 2 años, referidos por sus médicos gastroenterólogos tratantes y sometidos a colonoscopia izquierda por rectorragia. Se revisaron los registros de colonoscopias izquierdas realizadas en menores de 2 años con rectorragia, entre enero de 2006 y marzo de 2011 y luego los informes anatomopatológicos de las muestras tomadas durante esas colonoscopias, identificando aquellos pacientes en quienes la biopsia fue compatible con $\mathrm{CE}$.

\section{Pacientes y recolección de datos}

Se definieron como casos aquellos lactantes referidos por sus médicos tratantes a colonoscopia izquierda por antecedente de rectorragia de cualquier magnitud, en forma ambulatoria, cuya biopsia fuera compatible con el diagnóstico histológico de $\mathrm{CE}$, definida como el hallazgo de más de 60 eosinófilos por 10 CMA. Se definieron como controles aquellos lactantes referidos por sus médicos tratantes a colonoscopia izquierda por antecedente de rectorragia de cualquier magnitud, en forma ambulatoria, cuya biopsia no cumpliera los criterios histológicos antes referidos.

\section{Cuestionario clínico}

A las madres y/o padres de todos los niños en quienes se realizó este procedimiento, se les contactó telefónicamente y se les explicó los objetivos del estudio. Se les comunicó acerca de la confidencialidad del estudio e invitó a participar voluntariamente. A quienes aceptaron, se les realizó un cuestionario, también vía telefónica. Esta herramienta es un cuestionario local, diseñado para este estudio y sólo usado en este centro. Constó de 25 preguntas, tardó aproximadamente 5 minutos en aplicarse e incluyó antecedentes personales del paciente (prematurez, peso de nacimiento), edad de inicio de la rectorragia, síntomas que acompañaron a la rectorragia, otras enfermedades asociadas a $\mathrm{CE}$ y el tipo de alimentación recibida. Además se investigó la presencia de enfermedades asociadas a CE en familiares de primer grado. Se intentó contactarlos hasta con 10 intentos, luego de lo cual se los eliminó del listado.

\section{Análisis estadístico y aspectos éticos}

Las variables categóricas fueron descritas como número y porcentaje. Las variables numéricas como mediana y rango intercuartil. Para la comparación entre variables categóricas se utilizó el Test Exacto de Fisher, y para la comparación entre variables categóricas y numéricas se utilizó el Test de Mann-Whitney. Se consideró estadísticamente significativo todo valor $\mathrm{p}$ menor a 0,05 . Los análisis fueron realizados mediante el software estadístico SPSS versión 15.0.

Este proyecto fue aprobado por el Comité de Ética de la institución albergante de acuerdo a las normas vigentes previo al inicio del estudio.

\section{Resultados}

\section{Pacientes}

Durante el período descrito, se realizaron 77 colonoscopias izquierdas (hasta el ángulo esplénico del colon) por rectorragia recurrente o persistente. Se logró obtener datos y contactar a 61 pacientes $(79 \%)$. De estos, $33(54 \%)$ eran hombres. La edad promedio de realización del examen fue de 6,3 $\pm 5,9$ meses. De ellos, 25(41\%) pacientes presentaron CE y por lo tanto, se consideraron casos y $36(59 \%)$ pacientes tuvieron biopsias no compatibles con CE (controles). En estos casos no se logró evidenciar la causa de la rectorragia. La edad promedio de realización del examen para los casos fue de 4,6 meses (1-15 meses) y la de los controles de 7,5 meses (1-23 meses). En 13 de los pacientes se realizó la colonoscopia como parte del diagnóstico diferencial y en el resto fue realizada por falta de respuesta a tratamiento.

\section{Variables clínicas}

Como se aprecia en la tabla 1, al comparar los datos entre aquellos pacientes que tuvieron 
diagnóstico histológico de CE (casos) y quienes no lo tuvieron (controles), no hubo diferencia significativa en ninguna de las variables salvo en la edad de presentación de la rectorragia $(p=0,001)$, siendo de 1,8 meses en promedio para los con CE positiva y de 5 meses promedio para los con CE negativa (tabla 2 ).

\section{Antecedentes personales}

Los síntomas más frecuentes asociados a la rectorragia entre los niños con CE fueron diarrea $(\mathrm{p}=0,205)$, cólicos $(\mathrm{p}=0,610)$, e irritabilidad $(p=1,0)$, no diferenciándose significativamente del grupo control (tabla 2). Tampoco hubo diferencias significativas entre ambos grupos en cuanto a síntomas como reflujo gastroesofágico $(\mathrm{p}=0,505)$ o constipación $(\mathrm{p}=0,725)$.

Aquellos pacientes con antecedentes anamnésticos según sus padres de bronquitis obs- tructiva recurrente, Rinitis alérgica $u$ otras alergias, ya sea alimentarias, medicamentosas o a picaduras de insecto, que se presentaron entre el momento de la colonoscopia y la entrevista telefónica, no tuvieron mayor frecuencia de CE comparado con aquellos que no presentaron ninguno de estos síntomas (tabla 1).

\section{Antecedentes familiares}

Dentro de los antecedentes familiares, no hubo diferencia significativa de asma, rinitis alérgica, dermatitis atópica, anafilaxia ni alergias alimentarias, medicamentosas o a picaduras de insectos, entre familiares de casos y controles (tabla 3).

\section{Colonoscopia}

Los hallazgos más frecuentes en este examen fueron las erosiones $(19,7 \%)$ y la nodularidad $(72,1 \%)$, sin existir diferencias signifi-

Tabla 1. Características biodemográficas

\begin{tabular}{|c|c|c|c|c|}
\hline Características & $\begin{array}{l}\text { CE Positiva } \\
n=25\end{array}$ & $\begin{array}{l}\text { CE Negativa } \\
n=36\end{array}$ & $\begin{array}{l}\text { Total } \\
n=61\end{array}$ & $\mathbf{p}$ \\
\hline Sexo, masc/fem (\%) & $15 / 10(60 / 40)$ & 18/18 (50/50) & $33 / 28(54 / 46)$ & NS \\
\hline Prematuros, n (\%) & $5(20)$ & $4(11,1)$ & $9(14,7)$ & NS \\
\hline Peso de nacimiento, $\mathrm{g}$ & 3.305 & 3.262 & 3.280 & NS \\
\hline $\begin{array}{l}\text { Pacientes con antecedentes de*: } \\
\text { SBOR, } \mathrm{n}(\%)\end{array}$ & $5(20)$ & $6(16,6)$ & $11(18)$ & NS \\
\hline Rinitis alérgica, $n(\%)$ & $10(40)$ & $21(58,3)$ & $31(50)$ & NS \\
\hline Otras alergias alimentarias, n (\%) & $6(24)$ & $9(25)$ & $15(24,5)$ & NS \\
\hline Alergias a medicamentos, $\mathrm{n}(\%)$ & $2(8)$ & $1(2,7)$ & $3(4,9)$ & NS \\
\hline Alergias picaduras de insectos, n (\%) & $2(8)$ & $8(22,2)$ & $10(16,4)$ & NS \\
\hline
\end{tabular}

*Por cuestionario.

Tabla 2. Síntomas asociados a la rectorragia

\begin{tabular}{|c|c|c|c|c|}
\hline Características & $\begin{array}{c}\text { CE Positiva } \\
n=25\end{array}$ & $\begin{array}{c}\text { CE Negativa } \\
n=36\end{array}$ & $\begin{array}{c}\text { Total } \\
n=61\end{array}$ & $\mathbf{p}$ \\
\hline Inicio rectorragia, promedio meses & 1,8 & 5 & 3,7 & 0,001 \\
\hline Diarrea, n (\%) & $11(44)$ & $22(61,1)$ & $33(54)$ & NS \\
\hline RGE, n (\%) & $3(12)$ & $7(19,4)$ & $10(16,4)$ & NS \\
\hline Constipación, n (\%) & $3(12)$ & $6(16,7)$ & $9(14,8)$ & NS \\
\hline Cólicos, n (\%) & $13(52)$ & $16(44,4)$ & $29(47,5)$ & NS \\
\hline Irritabilidad, n (\%) & $9(36)$ & $13(36,1)$ & $22(36)$ & NS \\
\hline Letargia, n (\%) & 0 & $5(13,8)$ & $5(8,2)$ & NS \\
\hline Síntomas cutáneos, n (\%) & $4(16)$ & $7(19,4)$ & $11(18)$ & NS \\
\hline
\end{tabular}


Tabla 3. Antecedentes familiares de los pacientes en estudio

\begin{tabular}{|c|c|c|c|c|}
\hline n familiares con: & $\begin{array}{c}\text { CE Positiva } \\
n=25\end{array}$ & $\begin{array}{c}\text { CE Negativa } \\
n=36\end{array}$ & $\begin{array}{c}\text { Total } \\
n=61\end{array}$ & $\mathbf{p}$ \\
\hline Asma, n (\%) & $7(28)$ & $8(22,2)$ & $15(24,6)$ & NS \\
\hline Rinitis alérgica, n (\%) & $12(48)$ & $22(61,1)$ & $34(55,7)$ & NS \\
\hline Dermatitis atópica, n (\%) & $7(28)$ & $14(38,9)$ & $21(34,4)$ & NS \\
\hline Anafilaxia, n (\%) & $5(20)$ & $4(11,1)$ & $9(14,8)$ & NS \\
\hline Otras alergias alimentarias, n (\%) & $3(12)$ & $6(16,7)$ & $9(14,8)$ & NS \\
\hline Alergias a medicamentos, $\mathrm{n}(\%)$ & $6(24)$ & $10(27,8)$ & $16(26,2)$ & NS \\
\hline Alergias a picaduras de insectos, $n(\%)$ & $7(28)$ & $12(33,4)$ & $19(31,1)$ & NS \\
\hline
\end{tabular}

Tabla 4. Hallazgos en colonoscopía izquierda

\begin{tabular}{|lcccc|}
\hline Características & $\begin{array}{c}\text { CE Positiva } \\
\mathbf{n = 2 5}\end{array}$ & $\begin{array}{c}\text { CE Negativa } \\
\mathbf{n = 3 6}\end{array}$ & $\begin{array}{c}\text { Total } \\
\mathbf{n = 6 1}\end{array}$ & p \\
Edad colonoscopía izquierda, meses & 4,8 & 7,6 & 6,5 & NS \\
n pacientes con: & & & & \\
Colonoscopía normal, n (\%) & $1(4)$ & $6(16,7)$ & $7(11,5)$ & NS \\
Eritema, n (\%) & $2(8)$ & $2(5,6)$ & $4(6,6)$ & NS \\
Erosiones, n (\%) & $7(28)$ & $5(13,9)$ & $12(19,7)$ & NS \\
Úlceras, n (\%) & $4(16)$ & $3(8,3)$ & $7(11,5)$ & NS \\
Nódulos, n (\%) & $19(76)$ & $25(69,4)$ & $44(72,1)$ & NS \\
Sangre visible, n (\%) & $2(8)$ & $1(2,8)$ & $3(4,9)$ & NS \\
Pérdida del patrón vascular, n (\%) & $5(20)$ & $6(16,7)$ & $11(18)$ & NS \\
Extensión hasta ángulo esplénico, n (\%) & $21(84)$ & $31(86,1)$ & $52(85,2)$ & \\
\hline
\end{tabular}

cativas en estos hallazgos macroscópicos entre ambos grupos (eritema $\mathrm{p}=0,051$ y nodularidad $\mathrm{p}=0,759)($ tabla 4$)$.

\section{Exámenes de laboratorio complementarios}

A $33(54 \%)$ de los pacientes se les realizó estudio con IgE específica para leche de vaca y a 12 niños $(20 \%)$, se les realizó test cutáneos para antígenos alimentarios, solicitados por sus médicos tratantes previos a la colonoscopia, sin encontrar diferencias entre casos y controles.

\section{Discusión}

La rectorragia es la principal manifestación de la CE. Sin embargo, el síntoma, en forma aislada es inespecífico ${ }^{8,9}$. El Gold standard para el diagnóstico de $\mathrm{CE}$ es el test de provocación oral doble ciego ${ }^{11-13}$, examen con requerimiento de personal entrenado y no disponi- ble fácilmente. Se podría considerar el realizar exámenes de ciego simple, recordando que estos no deben realizarse en pacientes que han presentado anafilaxia. Al ser la CE una entidad no mediada por IgE, no existen exámenes de laboratorio no invasivos que permitan establecer el diagnóstico con mayor certeza. Es necesario por lo tanto, considerar la historia personal y familiar e incluso en casos seleccionados realizar exámenes endoscópicos e histológicos, para evitar tratamientos empíricos, muchas veces innecesarios.

Este estudio apoya la importancia de considerar diversas etiologías frente a un lactante con rectorragia. A pesar del sesgo inherente de que los pacientes referidos a estudio endoscópico pudieran estar seleccionados, en nuestro estudio de los 61 lactantes estudiados con colonoscopia izquierda por rectorragia, sólo un $41 \%$ tenían histología compatible con CE. Xanthakos et al, usando los mismos criterios para el diagnóstico de $\mathrm{CE}$ en 22 lactantes me- 
nores de 6 meses, obtuvieron una prevalencia de $\mathrm{CE}$ de $64 \%{ }^{8}$. En el resto de los pacientes del estudio no se logró precisar etiología.

La literatura establece que más de un tercio de los pacientes con dermatitis atópica moderada a severa, presentan reactividad a proteínas alimentarias ${ }^{18-20}$. En nuestro estudio, no hubo diferencia en cuanto a APLV entre aquellos niños cuyos padres referían diagnóstico de Dermatitis atópica y quienes no lo presentaban. Otro hallazgo importante es que el 50\% de los pacientes habría tenido diagnóstico de Rinitis alérgica según lo señalado por los padres, sin diferencias entre casos y controles. Además de las limitaciones propias de un cuestionario telefónico, como es el sesgo de memoria, estos resultados podrían explicarse debido a que los pacientes de este estudio aún no han desarrollado completamente los síntomas de la marcha atópica, por lo que alguno de ellos podría presentar atopía posteriormente. Así mismo, se describe un mayor riesgo de desarrollar alergia en aquellos con familiares atópicos. Diversos estudios muestran que el tener un padre, madre o hermano atópico aumenta el riesgo de este tipo de alergia en un 20 a un $40 \%$. Si ambos padres son atópicos, este se incrementa hasta en un $60 \% \%^{2,21,22}$. En nuestro estudio, 21 pacientes $(34,4 \%)$ tenían alguno de los padres o hermanos que refería diagnóstico de Dermatitis atópica. Siete de estos tenían CE y 14 de estos no tenían CE, lo cual iría en contra de lo previamente publicado. Además, $55,7 \%$ de los familiares refieren diagnóstico de Rinitis alérgica y $24,6 \%$ de Asma bronquial, sin diferencia entre ambos grupos. Esto resulta una proporción muy elevada, sin embargo, se ha demostrado que la estimación de alergia por la población está sobrevalorada, por lo cual no podemos asegurar que todos aquellos parientes que dicen ser alérgicos, lo son realmente ${ }^{6}$.

Los síntomas de las alergias alimentarias se presentan a edades tempranas, siendo la mayoría de ellos gastrointestinales. En el estudio los pacientes presentaron rectorragia a una edad promedio de 4,4 meses, siendo menor la edad de presentación de aquellos con CE (con significancia estadística). Los síntomas más comunes, tanto en el grupo estudiado, como en el control, fueron diarrea, cólicos e irritabilidad, síntomas frecuentes y difíciles de establecer a esta edad. Dentro de los pocos trabajos nacionales respecto al tema se encuentra el de la Dra. Cruchet ${ }^{23}$ en el cual estudian 49 pacientes entre 2 y 83 meses con diagnóstico de APLV por medio de contrapruebas orales. En estos pacientes, sólo un $10 \%$ presentó rectorragia. Sin embargo, los síntomas más comunes fueron diarrea, vómitos y dolor abdominal, similar a lo encontrado en nuestro estudio.

Si bien se ha sugerido una relación entre APLV y reflujo gastroesofágico en hasta un $50 \%{ }^{24-27}$, esto es discutido y puede ser necesario considerar su asociación en reflujo gastroesofágicos severos o que no responden a los tratamientos habituales. A su vez, existen estudios que muestran asociación entre APLV y constipación, sin embargo, esta relación está menos documentada ${ }^{28,29}$. En la población estudiada, no hubo mayor prevalencia de reflujo ni constipación entre los pacientes con $\mathrm{CE}$ en comparación a los sin CE.

La colonoscopia permite apreciar la mucosa colónica, siendo los hallazgos poco específicos de CE. Los más frecuentes son eritema, erosiones y en algunos casos úlceras. La presencia de nodularidad ha sido discutida, resultando inespecífica ${ }^{14,15,30}$. En el estudio los hallazgos más comunes en aquellos con $\mathrm{CE}$ fueron el eritema y la nodularidad, sin haber diferencia significativa con aquellos que no presentaron CE.

Es importante destacar que a pesar de que estudios de IgE específica y test de parche no estarían indicados en este tipo de alergia ${ }^{31}$, por no ser esta mediada por IgE y por sólo medir sensibilización, más de la mitad de los pacientes tenía estos exámenes realizados previos a la colonoscopia, no existiendo diferencia significativa entre casos y controles.

Según la literatura, la prevalencia de CE sería mayor en aquellos lactantes alimentados con fórmulas lácteas y la lactancia materna ejercería una protección contra las alergias alimentarias ${ }^{4,5}$. En nuestro estudio no se pudo evidenciar esta asociación, ya que aquellos alimentados con leche materna no tuvieron menor riesgo de tener $\mathrm{CE}$ que aquellos alimentados con fórmula artificial.

La prevalencia de CE en niños estudiados 
con colonoscopia izquierda por rectorragia persistente fue de $40,9 \%$. No hubo asociación con antecedentes personales ni familiares de atopía ni diferencia entre los tipos de alimentación que estos pacientes recibían. Los hallazgos endoscópicos fueron inespecíficos. Estos datos no se correlacionan estrictamente con lo publicado previamente, en cuanto a la importancia de la historia y antecedentes en el diagnóstico. Esto podría deberse a las limitaciones propias del estudio, ya que debido a su naturaleza observacional, los casos y controles pudieron diferir en otras características no exploradas, las cuales serían responsables del sesgo de confusión; asimismo, la evaluación retrospectiva de la exposición lo hace susceptible de presentar sesgo de memoria. Por otro lado, existe la posibilidad que resultados no significativos se expliquen por una falta de potencia estadística debido a la baja prevalencia de esta patología y no por una real ausencia de diferencia. Estos elementos motivan a plantear la necesidad de realizar estudios de tipo prospectivo y multicéntricos, los cuales permitirán reducir la ocurrencia de sesgos y aumentar el número de pacientes analizados, hecho especialmente importante al momento de estudiar enfermedades poco frecuentes a nivel poblacional, como es la CE.

Es importante ante la sospecha de APLV realizar suspensión del alérgeno y contraprueba, como establece la guía sobre APLV del Ministerio de salud de Chile $^{36}$ (elaborada por un grupo de expertos de la Rama Chilena de Gastroenterología Pediátrica) y publicadas como revisión ${ }^{37}$.

Si bien el diagnóstico de la APLV se realiza la mayor parte de las veces mediante la historia clínica, antecedentes personales, familiares y respuesta a dietas de exclusión y contraprueba, nuestros resultados muestran que hay grupos de pacientes en los cuales no existen antecedentes de la historia familiar ni personal que permitan establecer claramente que el diagnóstico de rectorragia como manifestación de una CE. En casos seleccionados (sangrado persistente o recurrente), como es el caso de los pacientes de nuestro estudio, se pueden realizar exámenes endoscópicos a manera de establecer un oportuno y correcto diagnóstico de aler- gias alimentarias. De esta forma se logra tratar correctamente a aquellos afectados, no se realizan dietas restrictivas con riesgo nutricional y de alto costo a quienes no las necesiten y no se retrasa la detección de otras enfermedades.

Potenciales conflictos de interés: Este trabajo cumple con los requisitos sobre consentimiento/asentimiento informado, comité de ética, financiamiento, estudios animales y sobre la ausencia de conflictos de intereses según corresponda.

\section{Referencias}

1.- Sampson HA, Buckley RH, Metcalfe DD: Food Allergy. JAMA 1987; 258: 2886-90.

2.- Johansson S, Hourihane J, Bousquet J, et al: A revised nomenclature for allergy. Allergy 2001; 56: 813-24.

3.- Brill H: Approach to milk protein allergy in infants. Can Fam Physician 2008; 54: 1258-64.

4.- American Gastroenterological Association Clinical Practice and Practice Economics Committee. AGA Technical Review on the Evaluation of Food Allergy in Gastrointestinal Disorders. Gastroenterology 2001; 120: 1026-40.

5.- Vandenplas $Y$, Brueton $M$, Dupont $C$, et al: Guidelines for the diagnosis and management of cow's milk protein allergy in infants. Arch Dis Child 2007; 92: 902-8.

6.- Rona RJ, Keil T, Summers C, et al: The prevalence of food allergy: a meta-analysis. J Allergy Clin Immunol 2007; 120: 638-46.

7.- Martorell A, Plaza A, Boné J, et al: Cow's milk protein allergy. A multi-centre study: clinical and epidemiological aspects. Allergol and Immunopatol 2006; 34 (2): 46-53.

8.- Xanthakos S, Schwimmer J, Melin-Aldana H, Rothen$\operatorname{berg} M$, Witte D, Cohen M: Prevalence and Outcome of Allergic Colitis in Healthy Infants with Rectal Bleeding: A Prospective Cohort Study. J Pediatr Gastroenterol Nutr 2005; 41: 16-22.

9.- Arvola T, Ruuska T, Keranën J, Hyöty H, Salminen S, Isolauri E: Rectal bleeding in infancy: Clinical, Allergological and Microbiological Examination. Pediatrics 2006; 117 (4) e760-9.

10.- Cannioto Z, Berti I, Nartelossi S, et al: IBD and IBD mimicking enterolociltis in children younger than 2 years of age. Eur J Pediatr 2009; 168: 149-55.

11.- Sicherer S: Food Allergy. Lancet 2002; 360: 701-10.

12.- Bindslev-Jensen C, Ballmer-Weber B, Bengtsson, et al: Standarization of food challenges in patients with 
immediate reactions to foods-position paper from the European Academy of Allergology and Clinical Immunology. Allergy 2004; 59: 690-7.

13.- Niggemann B, Beyer K: Diagnosis of Food Allergy in Children: Toward a Standarization of Food Challenge. J Pediatr Gastroenterol Nutr 2007; 45: 399-404.

14.- Odze R, Wershil B, Leichtner A, Antonioli D: Allergic colitis in infants. J Pediatr 1995; 126 (2): 163-70.

15.- Lake A: Food-induced Eosinophilic Proctocolitis. J Pediatr Gastroenterol Nutr 2000; 30 (1): S58-60.

16.- American Gastroenterological Association Medical Position Statement: Guidelines for the Evaluation of Food Allergies. Gastroenterology 2001; 120: 102325.

17.- Arévalo F, Arias Stella J, Monge E, Arias Stella J: Biopsia de colon: Características histológicas en diferentes tipos de colitis crónica. Rev Gastroenterol Perú 2008; 28: 140-9.

18.- Eigenmann P, Sicherer S, Borkowski T, Cohen B, Sampson H: Prevalence of IgE-Mediated Food Allergy Among Children With Atopic Dermatitis. Pediatrics 1998; 101 (3): e8.

19.- Burks A, James J, Hiegel A, et al: Atopic dermatitis and food hypersensitivity reactions. J Pediatr 1998; 132: 132-6.

20.- Traube C, Ardelean-Jab D, Grimfeld A, Just J: La alergia alimenticia del niño. Acta Bioquímica Clín Latinoam 2004; 38 (3): 319-27.

21.- Beyer K, Teuber $S$ : Food allergy diagnostics: scientific and unproven procedures. Curr Opin Allergy Clin Immunol 2005; 5: 261-6.

22.- Herz U: Immunological Basis of Food Allergy. J Pediatr Gastroenterol Nutr 2008; 47: S54-57.

23.- Cruchet S, Faúndez R, Laguna C, Araya M: Contrapruebas en el diagnóstico y seguimiento de niños con alergia alimentaria. Rev Med Chile 2003; 131: 275-82.

24.- Iacono G, Carroccio A, Cavataio F, et al: Gastroesophageal reflux and cow's milk allergy: A prospective study. J Allergy Clin Immunol 1996; 97: 822-7.

25.- Cavataio F, Carroccio A, Iacono G: Milk-Induced Reflux in Infants Less Than One Year of Age. J Pediatr
Gastroenterol Nutr 2000; 30 Suppl: S36-44.

26.- Salvatore S, Vandenplas Y: Gastroesophageal Reflux and Cow Milk Allergy: Is There a Link? Pediatrics 2002; 110: 972-84.

27.- Nielsen R, Bindslev-Jensen C, Kruse-Andersen, Husby $S$ : Severe Gastroesophageal Reflux Disease and Cow Milk Hypersensitivity in Infants and Children: Disease association and Evaluation of a New Challenge Procedure. J Pediatr Gastroenterol Nutr 2004; 39: 383-91.

28.- Iacono G, Carroccio A, Cavataio F, Montalto G, Cantarero $M$, Notarbartolo A: Chronic constipation as a symptom of cow milk allergy. J Pediatr 1995; 126: 34-9.

29.- Iacono $G$, Cavatalo $F$, Montalto $G$, et al: Intolerance of Cow's Milk and Chronic Constipation in Children. NEJM 1998; 339 (16): 1100-4.

30.- Sampson H, Anderson J: Summary and Recommendations: Classification of Gastrointestinal Manifestations Due to Immunologic Reactions to Foods in Infants and Young Children. J Pediatr Gastroenteral Nutr 2000; 30: S87-S94.

31.- Bock A: Evaluation of Ig-Mediated Food Hypersensitivities. J Pediatr Gastroenterol Nutr 2000; 30: S20-7.

32.- Giampietro PG, Kjellman N, Oldaeus G, WoutersWesseling $W$, Businco L: Hypoallergenicity of an extensively hydrolyzed whey formula. Pediatr Allergy Immunol 2001; 12: 83-6.

33.- Cox H, Husby S: Debate: Current Challenges in the Management of Food Allergies. J Pediatr Gastroenterol Nutr 2008; 47: S53.

34.- Host A, Koletzko B, Dreborg S, et al: Dietary products used in infants for treatment and prevention of food allergy. Arch Dis Child 1999; 81: 80-4.

35.- Hill D, Murch S, Rafferty K, Wallis P, Green C: The efficacy of amino-acid based formulas in relieving the symptoms of cow's milk allergy: a systematic review. Clin Exp Allergy 2007; 37: 808-22.

36.- Guía clínica alergia a la proteína de la leche de vaca. Santiago: Minsal 2012. web.minsal.cl

37.- Miquel I, Arancibia E: Alergia a proteína de leche de vaca en el menor de un año. Rev Chil Pediatr 2012; 83 (1): 78-83. 